\title{
ASSESSMENT OF VIABILITY OF WHARTON'S JELLY MESENCHYMAL STEM CELLS ENCAPSULATED IN ALGINATE SCAFFOLD BY WST-8 ASSAY KIT
}

\author{
Zeinab Shafiei Seifabadi ${ }^{1 \dagger}$, Fatemeh Rezaei-Tazangi ${ }^{1 \dagger}$, Nastaran Azarbarz ${ }^{1}$, Darioush Bijan Nejad ${ }^{1,4}$, \\ Javad Mohammadiasl ${ }^{2}$, Hanieh Darabi ${ }^{3}$, Seyed Pezhmanlarki-Tork ${ }^{3}$
}

\begin{abstract}
Cell encapsulation utilizing biodegradable material has promising outcomes for tissue engineering. From a long time ago, alginate has been generally utilized for drug delivery, cell transplantation and as a scaffold in biomedical applications. The aim of this study was the comparison of cell viability in the presence of two polymerizing ions: $\mathrm{Ba}^{2+}$ and $\mathrm{Ca}^{2+}$ to improvement the quality of alginate scaffold. For this purpose, WJMSCs after three passage were encapsulated in alginate scaffold in the presence of $\mathrm{Ba}^{2+}$ and $\mathrm{ca}^{2+}$. Cell viability was evaluated by WST- 8 assay kit after 24, 48 and 72 hours. The results showed that encapsulated cells in the presence of $\mathrm{Ca}^{2+}$ had more viability than $\mathrm{Ba}^{2+}$. It was also found that using the WST- 8 assay kit is a convenient and fast method for evaluation the viability of cells. It can be claimed that Calcl2 polymerizing solution provides more favorable conditions for cell viability compared to Bacl2 solution.
\end{abstract}

Running title: Assessing the viability of stem cells by WST-8 assay kit

Keywords: Wharton's jelly, mesenchymal stem cells, alginate, viability

\footnotetext{
ॠZeinab Shafiei Seifabadi and Fatemeh Rezaei-Tazangi contributed equally to this work

${ }^{1}$ Department of Anatomical Sciences, Faculty of Medicine Ahvaz Jundishapur University of Medical Sciences, Ahvaz, Iran

${ }^{2}$ Department of Medical Genetics, Faculty of Medicine, Ahvaz Jundishapur University of Medical Sciences, Ahvaz, Iran

${ }^{3}$ Faculty of Medicine, Ahvaz Jundishapur University of Medical Sciences, Ahvaz, Iran

${ }^{4}$ Medical Basic Sciences Research Institute, Ahvaz Jundishapur University of Medical Sciences, Ahvaz, Iran

*Correspondence: daryoshbijan@yahoo.com

Full list of author information is available at the end of article
} 


\section{Introduction}

Mesenchymal stem cells (MSCs) are multipotent cells, which are able to self-renew and have high proliferative capacity. These characteristics of MSCs are considered to be very important for several applications in the field of regenerative medicine, including the development of cell-based therapies, tissue repair procedures and they are an ideal resource for tissue engineering applications. Alternative MSC sources are fetal tissues, extraembryonic tissues such as placenta and amniotic fluid, Umbilical cord, umbilical cord blood and stroma. Umbilical cord contains a special primitive connective tissue called Wharton's jelly, represents an interesting alternate source for MSCs [1].

Environmental stimulation are efficiently effect on stem cells. For example the path of the stem cell to self-renewal or differentiation is determined by the external signal. Cell encapsulation in hydrogels, such as alginate, is the challenge of scalable bioprocessing and enable suspension culture provide and better control of micro environmental condition [2,3]. Encapsulation has been used as a tool for enhancement of stem cell viability and differentiation. Alginate has been the most frequently used material, as it is non-toxic and the encapsulation can be carried out under physiological conditions. Mammalian cells encapsulation is commonly done with alginate, due to its mild cross-linking and relative abundance. Native alginate is non-adhesive and therefore cannot relate directly with cells, the interaction of alginate and enclosed cells depend on physical and chemical polymer properties [4].

Previous studies has been shown that encapsulated human embryonic stem cells on $1.1 \%(\mathrm{w} / \mathrm{v})$ calcium alginate hydrogels is maintain up to 260 days, without exposure to animal cells [5]. Various studies have been shown that MSCs inserted in alginate hydrogel represent a model for chondrogenic differentiation of human MSCs [6].

Fabrication and characterization of a scaffold are a key area of research for tissue engineering applications [7]. Natural derived polysaccharide such as alginate, widely used versatile biomaterials for bone tissue engineering applications [8]. Alginate is a $\mathrm{pH}$-sensitive, hydrophilic and biocompatible polysaccharide with the negative charge which is obtained from seaweed. It is composed of two monomers, $\beta$-D-mannuronate and $\alpha$-L-guluronate, known to form gels in the presence of bivalent cations such as calcium $\left(\mathrm{Ca}^{2+}\right)$. Given these characteristics, this polymer has been widely used for drug and growth factor delivery, cell encapsulation and as a scaffold in tissue engineering applications [9]. Depending upon the nature of the polymer, different techniques may be used to cause cross linking [10]. There is different methods for cross-linking of algi- nate scaffolds that is divided into two main physical and chemical methods. The cross-linking type and density have an influence on the mechanical feature of alginate. The physical cross-linking is anionic polymer chains can be connected by the divalent cations such as $\mathrm{Ca}^{2+}, \mathrm{Sr}^{2+}$ and $\mathrm{Ba}^{2+}$ form a structure of alginate similar to an egg-box [11]. Adding crosslinks between polymer chains affect the physical properties of the polymer depending upon the degree of cross linking and presence and absence of crystallinity. Cross linking leads to be elasticity, decline in the viscosity, insolubility, increase strength and stiffness, less melting point, transformation of therrmoplasts into thermosets [10].

The aim of this study was the proposing of optimal conditions to improve the viability and proliferation of WJMSCs in alginate beads. The paper describes the isolation method of human WJMSCs, the production of calcium (Ca)-alginate and barium (Ba)-alginate microbeads for WJMSCs encapsulation and at the end, the comparison of WJMSCs morphology in both types of alginate microbeads.

\section{Materials and Methods \\ WJMSCs: Isolation procedure and culture conditions}

Wharton's Jellies were collected from the infants born with cesarean incision. All protocols were approved by the Ethic Committee of Ahvaz Jundishapur University of Medical Sciences (approval number: IR.AJUMS.REC.1397.693) and in accordance with the university ethical guidelines. Informed consent forms were obtained from their parents. The umbilical cords were transferred to the laboratory in cold phosphate buffer saline (PBS) containing penicillin/streptomycin. The specimens were washed and flashed by PBS to remove the blood and the vein was cut longitudinally. The epithelium was scraped and the arteries were cut away. The rest of the umbilical cord, Wharton's jelly, was cut into small pieces. Each piece was mounted on the floor of a tissue culture plate. After 20 minutes, culture medium, Dulbecco's modified Eagle's medium (DMEM) containing $1 \%$ penicillin/streptomycin and $1 \%$ L-glutamine, was added to the explants for 10-15 days and cultured at $37^{\circ} \mathrm{C}, 5 \% \mathrm{CO} 2$. The culture media were replaced twice a week. The cells grown from the explants were preserved in the presence of DMEM containing $10 \%$ fetal bovine serum, $1 \%$ penicillin/ streptomycin, and 1\% L-glutamine [12]. After 5-7 days, the culture medium was removed and thereafter changed twice a week. After three passage, at a $70-80 \%$ confluence, cells were scraped off by $0.05 \%$ trypsin (Gibco, Grand Island, NE) (3 min), washed, counted by hemocytometric analysis, assayed for viability, and thereafter used for further in vitro experiments or for encapsulation procedures [1]. 


\section{WJMSCs characterization}

The WJMSCs were analyzed for expression of MSC surface marker molecules by flow cytometry technique. Briefly, cell pellets were re-suspended in PBS and incubated with anti-human antibodies against CD105, CD90, CD34, and CD45 (eBioscience, San Diego, CA) for $30 \mathrm{~min}$ at $4^{\circ} \mathrm{C}$ in the dark condition. Negative and isotype controls were performed. Antibody-treated cells were then washed with PBS. After cell staining, for each sample, 10,000 events were counted by a Dako Galaxy flow cytometer and data were analyzed using FlowJo version 8.8 .7 software (Treestar, OR). Osteogenic and adipogenic potential were evaluated using alizarin-red and oil red 0 staining respectively.

\section{Preparation of Alginate Hydrogels}

Preparation of alginate solutions with $1.8 \%(\mathrm{w} / \mathrm{v})$ concentration was performed by dissolving $450 \mathrm{mg}$ alginate, $219 \mathrm{mg} \mathrm{NaCl}$ and $115 \mathrm{mg}$ HEPES into deionized water. The solutions were heated on a hot plate and stirred them thoroughly. Their processes of filtration were sterilized using a $0.22-\mu \mathrm{m}$ filter.

Preparation of $\mathrm{CaCl}_{2}(1.2 \mathrm{mM})$ solution: $3 \mathrm{~g}$ of cacl2 and $0.476 \mathrm{~g}$ of Hepes was dissolved in $\mathrm{dH} 2 \mathrm{O}$ and sterilized using a $0.22-\mu \mathrm{m}$ filter.

Preparation of $\mathrm{BaCl}_{2}(1.2 \mathrm{mM})$ solution: Prepared the solution in $\mathrm{dH} 2 \mathrm{O}$ and sterilized using a $0.22-\mu \mathrm{m}$ filter. All the solutions were kept at $4^{\circ} \mathrm{C}$.

To polymerize the alginate, $50 \mu \mathrm{LaCl}_{2}$ and $\mathrm{BaCl}_{2}$ prepared in water was added to the bottom of a 24-well culture plate. The alginate solution was warmed at $37^{\circ} \mathrm{C}$, and $500 \mu \mathrm{L}$ was added to the $\mathrm{CaCl} 2$ and $\mathrm{BaCl}_{2}$ solutions. The plate was incubated for $30 \mathrm{~min}$ at room temperature and then for another $30 \mathrm{~min}$ at $4^{\circ} \mathrm{C}[13]$.

\section{Encapsulation of WJMSCs}

The WJ-MSCs were resuspended in the $1.8 \%$ alginate solutions at a concentration of $1 \times 10^{6}$ cells/ $\mathrm{ml}$. Then, they were mixed by slowly pipetting and drop falls from 22-gauge needle into the $\mathrm{CaCl}_{2}$ and $\mathrm{BaCl}_{2}$ solutions. The alginate beads were incubated at $37^{\circ} \mathrm{C}$ for $15 \mathrm{~min}$ to allow the Ca and Ba cations to fully diffuse through alginate and cross-link the alginate cell suspension. $\mathrm{CaCl}_{2}$ and $\mathrm{BaCl}_{2}$ solutions were pipetted off; after gelation, the alginate beads were washed several times with saline and the normal saline was replaced with $3 \mathrm{ml}$ of the culture medium (DMEM containing 10\% fetal bovine serum, $1 \%$ penicillin/streptomycin, and $1 \%$ L-glutamine) at $37^{\circ} \mathrm{C}$ in an humidified atmosphere of $5 \% \mathrm{CO}_{2}$.

After the encapsulation of cells, each of the trials was performed three times.

\section{Evaluation of cell viability with WST-8 assay}

The viability of the immobilized WJMSCs was measured by analyzing the enzymatic conversion of tetrazolium salt (WST-8 assay kit, Cayman, USA).
After an incubation time of 24,48 and $72 \mathrm{~h}, 10$ alginate beads in a 96-well plate in $500 \mu$ of culture medium without FBS was tested. $50 \mu$ l of the WST-8 Mixture was added to each well using a repeating pipette and gently for one minute, then was mixed on an orbital shaker. Alginate beads was kept in a $\mathrm{CO} 2$ incubator at $37^{\circ} \mathrm{C}$ for 2.5 hours. Before reading, the plate was mixed gently on an orbital shaker for one minute to ensure homogeneous distribution of color. The absorbance was measured of each sample using a microplate reader at a wavelength of $450 \mathrm{~nm}$.

\section{Statistical analysis}

All measurement data were expressed as the mean-standard deviation. The statistics generated in this study were performed using the SPSS software (version16.0, Chicago, IL, USA). Significant differences were analyzed by The Mann-Whitney U test. A level of $\mathrm{P}<0.05$ was considered statistically significant.

\section{Ethical approval}

The research related to human use has been complied with all the relevant national regulations, institutional policies and in accordance with the tenets of the Helsinki Declaration. It has been approved by Ahvaz Jundishapur University of Medical Sciences (AJUMS) Bioethical Committee with approval no. IR.AJUMS.REC.1397.693.

\section{Informed consent statement}

Informed consent has been obtained from all individuals included in this study.

\section{Results \\ Morphological assessments}

The primary cell buds were observed around each explant after 7-10 days. The morphology of HWJMSCs was fibroblastic at primary culture and first passage. After 3 passages the cells had many cytoplasmic processes (Fig. 1).

The Flow Cytometry showed that these cells had no expression for CD34 and CD144, while the expression of CD44 and CD90 was high (Fig. 2).

\section{Osteogenic and adipogenic differentiation}

The pluripotency of WJMSCs was evaluated by oil red and alizarin red staining. After exposure to osteogenic differentiation medium the $\mathrm{Ca}^{2+}$ crystals were observed in these cells and they could store lipid droplets in response to adipogenic differentiation (Fig. 3).

\section{The morphology of encapsulated cells}

The morphology of cells within the alginate beads were investigated by using light inverted microscope. At day 1 post encapsulation, the morphology of encapsulated cells was round. The cells appeared to obtain their original round shape during the time of the study. Numerous fine pro- 

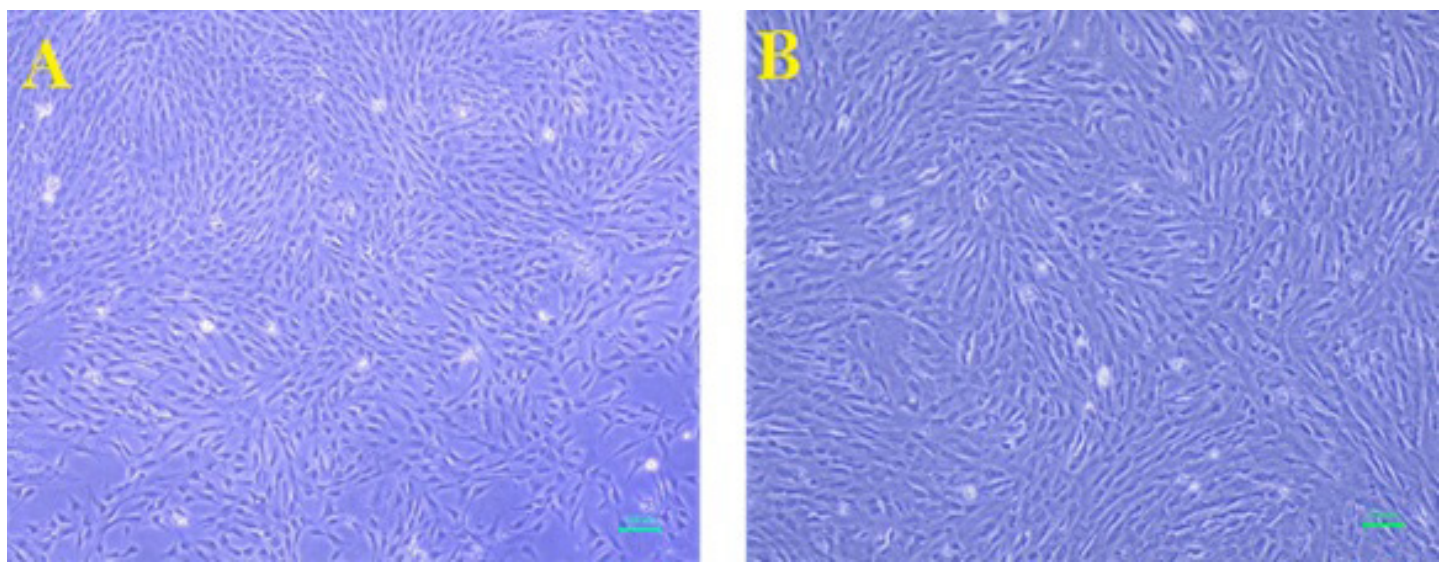

FIGURE 1 The Morphology of Wharton's Jelly-Derived MesenchymalStem Cells at (A) Primary Culture and (B) 3rd Passage
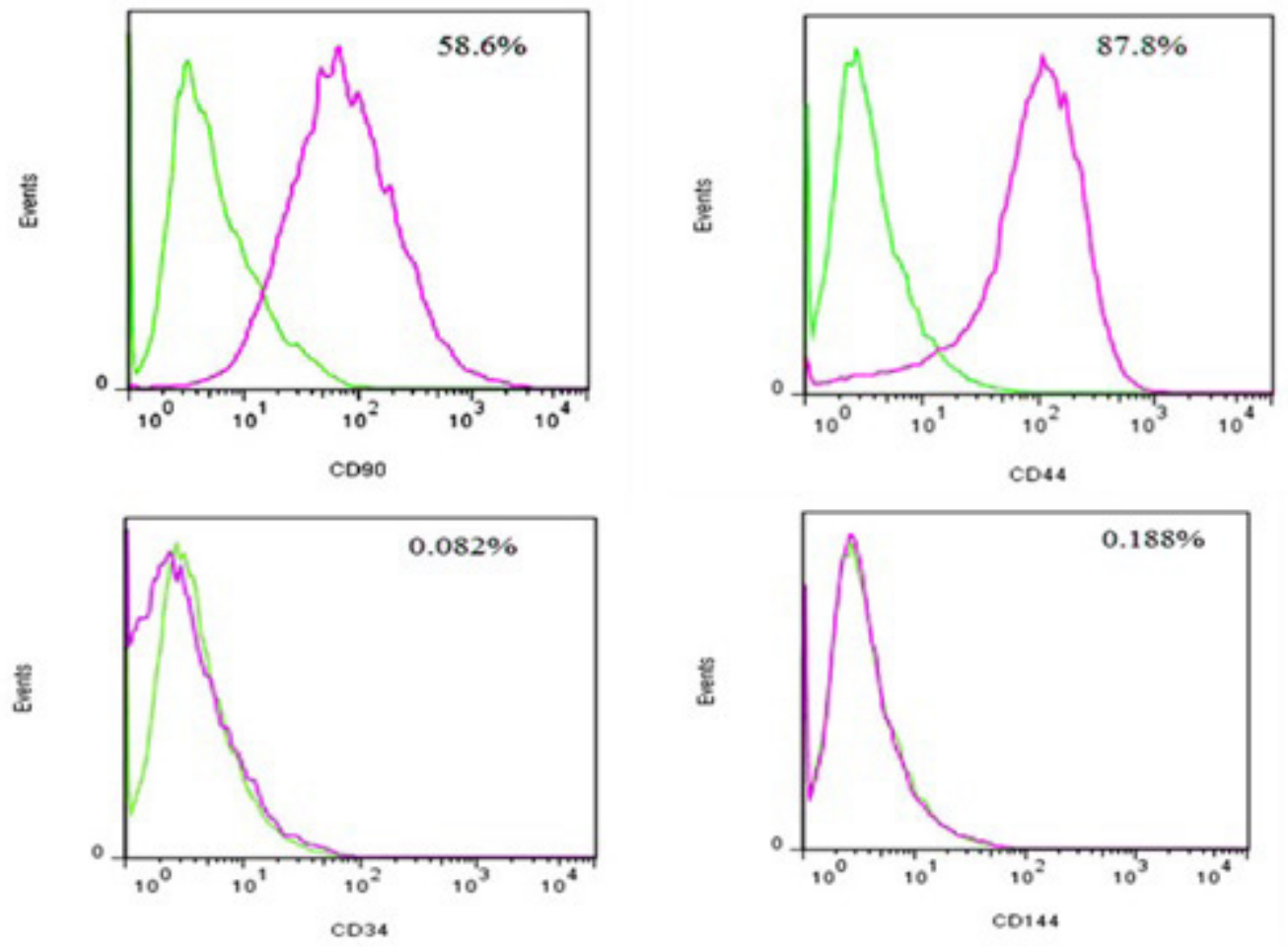

FIGURE 2 The Frequency of the Positive Cells for CD Markers by Flow Cytometry. The HUCWJ MSCs expressed CD44 and CD90, but they expressed CD34 and CD144 with less frequency
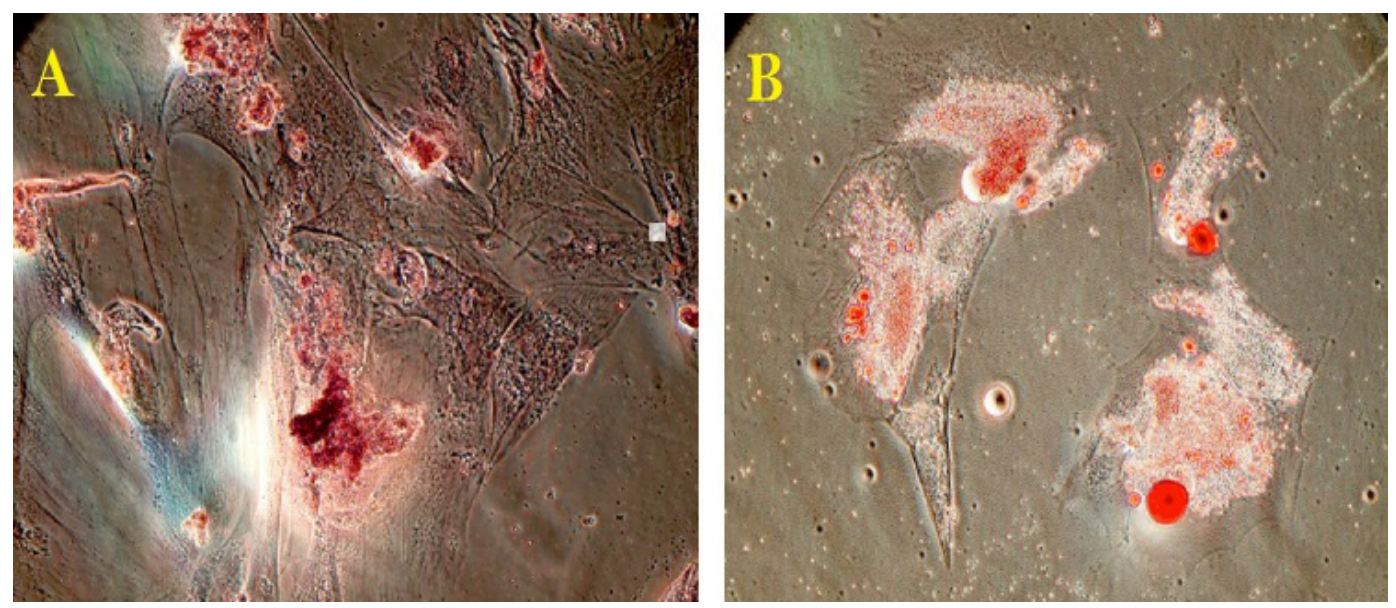

FIFURE 3 Osteogenic differentiation was confirmed by staining of Calcium deposits with Alizarin Red/S (A). Oil Red showed the presence of lipid droplets in the cells cultured in adipogenic medium (B). Magnification: $400 \mathrm{X}$ 

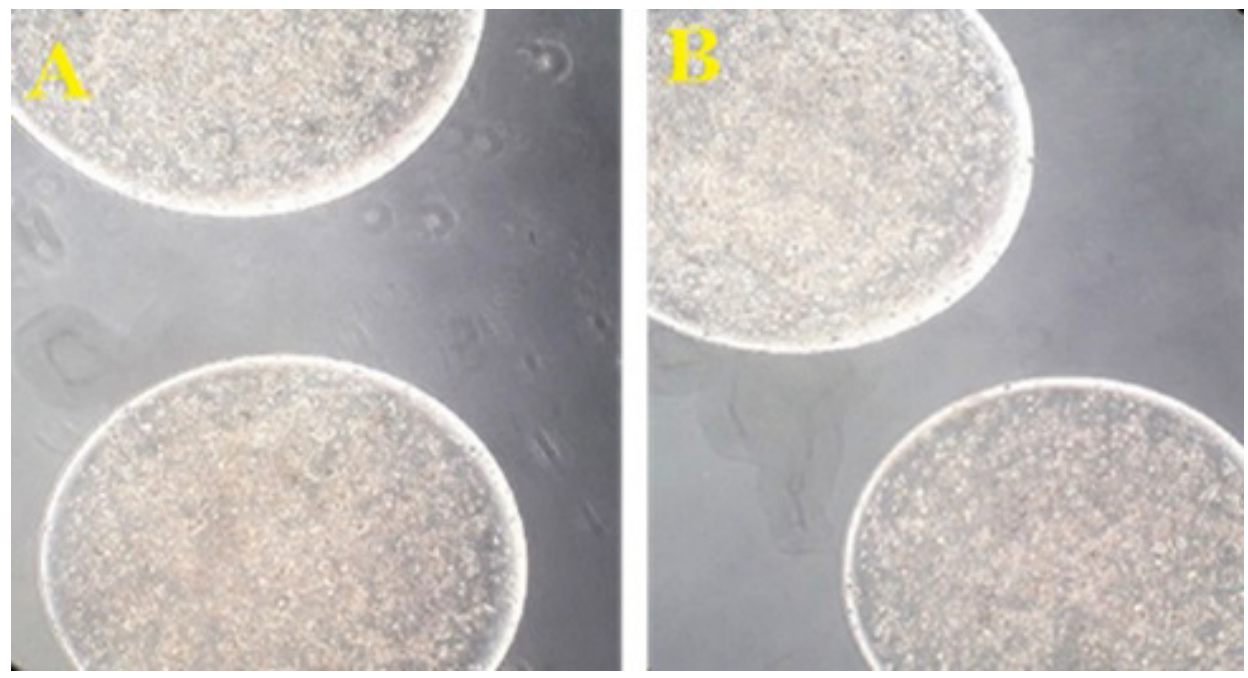

FIGURE 4 Morphology of the cells within the alginate beads. A. Alginate/Cacl2 B. Alginate/Bacl2. Magnification: 250 X

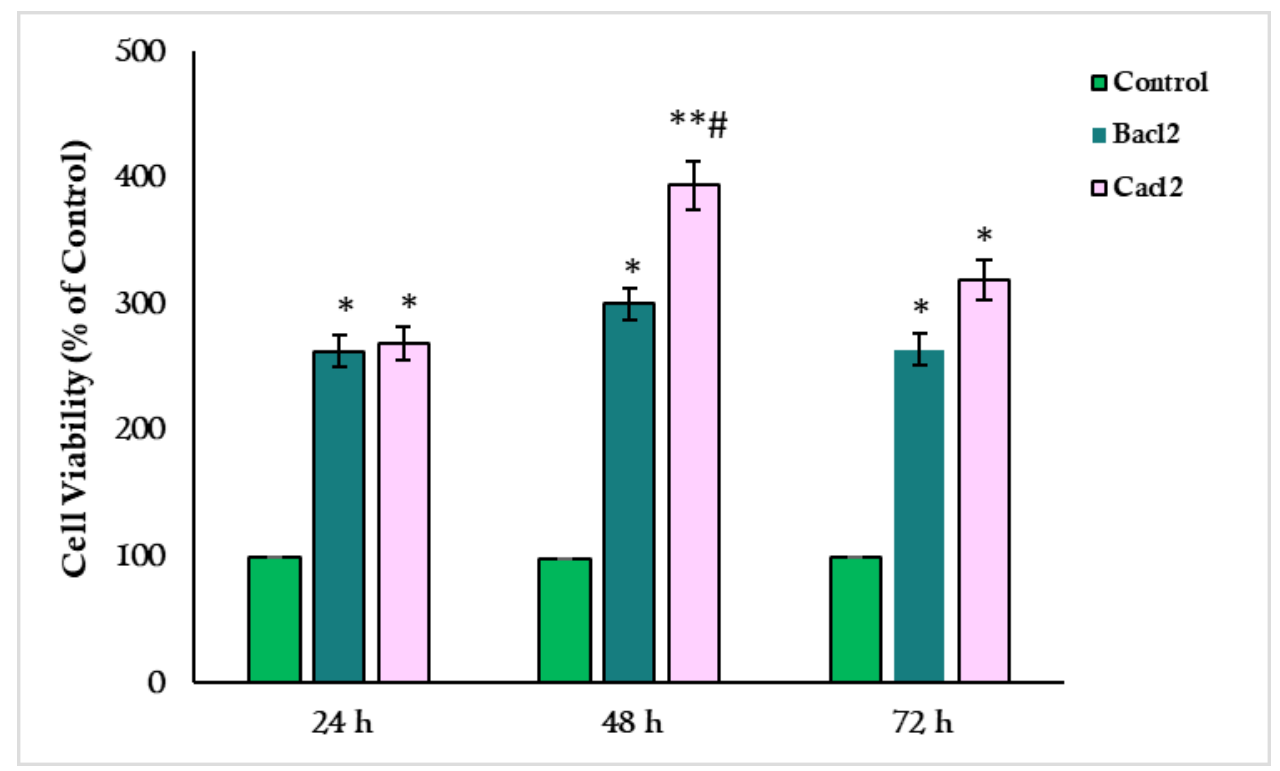

FIGURE 5 Cell viability within capsules after 24,48 and $72 \mathrm{~h}$. Values are expressed as mean $\pm \mathrm{SD}$. *; $\mathrm{P}<0.05$, **; $\mathrm{P}<0.01$, \#; P $<0.05 . *$ And \# symbols indicate comparison to the control and Bacl2 groups

cess spreading out of cells among matrix of alginate were observed (Fig. 4).

\section{Cell viability}

The viability of encapsulated cells was significantly more than control group. After 24 hours post encapsulation, no significant difference was observed between the viability of cells that were encapsulated in Cacl2 solution in comparison with Bacl 2 solution. After 48 and 72 hours, the cells that were encapsulated in cacl2, had more viability than those were encapsulated in Bacl2 solution ( $p<$ 0.05). After 48 hours post encapsulation, the viability percent was diminished (Fig. 5).

\section{Discussion}

Wharton's jelly (WJ) can be used an inexpensive and accessible stem cell source, it had not require invasive procedure in comparison with bone marrow MSCs and other sources. WJMSCs have many clinical applications because they don't cause immune rejection. Alginate as a natural polysaccharide can be easily modified to various structures and application. This natural hydrogel shows excellent biocompatibility and biodegradability with several unique properties that have enabled it to be used as a matrix for tissue engineering $[12,13]$. Alginate provides entrapping of cells under physiological conditions, ensuring cell viability and function. [14-17].

$\mathrm{Ba}^{2+}$ has in recent years frequently been used as crosslinking ion in alginate hydrogels for cell encapsulation purposes, especially for capsules without the use of a stabilizing polycation. [14-19]. Calcium chloride solution forms hydrogels under mild conditions and its mechanical strength can be well con- 
trolled by crosslinking for various intervals of time. Also calcium chloride crosslinked blends are widely preferred for biomedical applications, due to its nontoxicity and the films obtained are more compact in nature. Barium chloride solution is known for its strong cross linkage between polymer chains. Alginate forms strong gels with $\mathrm{Ba}^{2+}$ and $\mathrm{Sr}^{2+}$ ions. As observed in this experiment, the morphology of cells within three-dimensional alginate capsules were round and spherical, unlike two-dimensional culture that show spindle-like fibroblastic morphology for HUCMSCs which led to formation a network and interaction between them $[13,20]$. In this study, the viability of encapsulated cells as an essential factor in tissue engineering was investigated by WST-8 assay kit. One of the advantages of using this kit is that does not cause toxicity to cells, can be done easily, and is more sensitive and stable than MTT. MTT was the first used, developed in the 1980s along with the growing popularity of 96-well microplate assays. It is still widely used as the standard method to determine cell population and health, but since the MTT compound is cytotoxic and only remains stable for a few hours, other alternative assays have been developed.

Our result showed that the viability of cells could be enhanced by increasing time in both type of capsules till 48 hours. After that, the viability of encapsulated cells was decreased with the prolongation of encapsulating duration. The viability of cells in the presence of $\mathrm{Ca}^{2+}$ was more than $\mathrm{Ba}^{2+}$, it can may be related to toxic effects of barium.

\section{Conclusions}

The present study showed that Cacl 2 polymerizing solution provides more favorable conditions for alginate encapsulated cells than Bacl2 polymerizing solution.

\section{Acknowledgements}

This study funded by grant no. 9719, from the Cellular and Molecular Research Center (CMRC-9719), of the Ahvaz Jundishapur University of Medical Sciences (AJUMS), Ahvaz, Iran.

\section{Corresponding author}

Darioush Bijan Nejad, Cellular and Molecular Research Center, Faculty of Medicine, Ahvaz Jundishapur University of Medical Sciences, Ahvaz, Iran. Tel: +98-611-3337370, Fax: +98-611-3337370, e-mail: daryoshbijan@yahoo.com

\section{Conflict of interest statement}

The authors declare they have no conflict of interest.

\section{References}

1. Penolazzi L, Tavanti E, Vecchiatini R, Lambertini E, Vesce F, Gambari R, et al. Encapsulation of mesenchymal stem cells from Wharton's jelly in alginate microbeads. Tissue Eng. Part C Methods. 2009;16(1):141-55; DOI: $10.1089 /$ ten.TEC.2008.0582.

2. Wilson JL, Najia MA, Saeed R, McDevitt TC. Alginate encapsulation parameters influence the differentiation of microencapsulated embryonic stem cell aggregates. Biotechnol Bioeng. 2014;111(3):618-31; DOI: org/10.1002/bit.25121.

3. Reppel L, Schiavi J, Charif N, Leger L, Yu H, Pinzano A, et al. Chondrogenic induction of mesenchymal stromal/stem cells from Wharton's jelly embedded in alginate hydrogel and without added growth factor: an alternative stem cell source for cartilage tissue engineering. Stem cell res. Ther. 2015;6(1):260; DOI: 10.1186/s13287-015-0263-2.

4. Gupta P, Nayak KK. Optimization of keratin/alginate scaffold using RSM and its characterization for tissue engineering. Int J Biol Macromol. 2016;85:141-9; DOI:org/10.1016.

5. Venkatesan J, Bhatnagar I, Manivasagan P, Kang K-H, Kim S-K. Alginate composites for bone tissue engineering: a review. Int J Biol Macromol. 2015;72:269-81; DOI: 10.1016/j.ijbiomac.2014.07.008.

6. Barros J, Ferraz MP, Azeredo J, Fernandes M, Gomes P, Monteiro F. Alginate-nanohydroxyapatite hydrogel system: Optimizing the formulation for enhanced bone regeneration. Mater Sci Eng C. 2019;105:109985; DOI: org/10.1016/j.msec.2019.109985.

7. Maitra J, Shukla VK. Cross-linking in hydrogels-a review. Am J Polym Sci. 2014;4(2):25-31; DOI:10.5923/j.ajps.20140402.01.

8. Farokhi M, Jonidi Shariatzadeh F, Solouk A, Mirzadeh H. Alginate Based Scaffolds for Cartilage Tissue Engineering: A Review. Int J Polym Mater Polym Biomate. 2019:1-18; DOI: 10.1080/00914037.2018.1562924.

9. Bahmanpour S, Khozani TT, Tazangi FR. Evaluation of the Capability of the Wharton's Jelly Mesenchymal Stem Cell Aggregates to Express the Markers of Three Germ Cell Lineages. Arch Iran Med. (AIM). 2019;22(2):85-90; DOI: 85064840517.

10. Hosseini SM, Vasaghi A, Nakhlparvar N, Roshanravan R, Talaei-Khozani T, Razi Z. Differentiation of Wharton's jelly mesenchymal stem cells into neurons in alginate scaffold. Neur Reg Res. 2015;10(8):1312; DOI: 10.4103/1673-5374.162768.

11. Mata M, Milian L, Oliver M, Zurriaga J, Sancho-Tello M, Llano JJMd, et al. In vivo articular cartilage regeneration using human dental pulp stem cells cultured in an alginate scaffold: a preliminary study. Stem cells Int. 2017;2017; DOI: org/10.1155/2017/8309256.

12. Bijan Nejad D, Azandeh S ,Habibi R, Mansouri E, Bayati V, Ahmadi Angal $\mathrm{K}$. Investigation of the role of alginate containing high guluronic acid on osteogenic differentiation capacity of human umbilical cord Wharton's jelly mesenchymal stem cells. J Microencapsul. 2017;34(8):732-743; DOI: org/10.1080/02652048.2017.1393115.

13. Morshed M, Mallick J, Nath AK, Uddin Z ,Dutta M, Hossain A, et al. Effect of barium chloride as a cross linking agent on the sodium alginate based diclofenac sodium beads. Bengladesh Pharm J. 2012;15(1):53-7; DOI: 55735232.

14. Mørch YA, Qi M, Gundersen POM, Formo K, Lacik I, Skjåk-Bræk G, et al. Binding and leakage of barium in alginate microbeads. J Biomed Mater Res A. 2012;100(11):2939-47; DOI: org/10.1002/jbm.a.34237.

15. Wilson JL, Najia MA, Saeed R, McDevitt TC. Alginate encapsulation parameters influence the differentiation of microencapsulated embryonic stem cell aggregates. Biotechnol Bioeng. 2014;111(3):618-31; DOI: 10.1002/bit.25121.

16. Moya ML, Morley M, Khanna O, Opara EC, Brey EM. Stability of alginate microbead properties in vitro. J Mater Sci Mater. Med. 2012;23(4):90312; DOI: $10.1007 / \mathrm{s} 10856-012-4575-9$.

17. Sun J, Tan H. Alginate-based biomaterials for regenerative medicine applications. Materials. 2013;6(4):1285-309; DOI: 10.3390/ma6041285.

18. Qi M, Strand BL, Morch Y, Lacik I, Wang Y, Salehi P, Barbaro B, Gangemi A Kuechle J, Romagnoli T, et al. Encapsulation of Human Islets in Novel Inhomogeneous Alginate- $\mathrm{Ca}^{2+} / \mathrm{Ba}^{2+}$ Microbeads: In Vitro and In Vivo Function. Artif Cell Blood Substit Immobil Biotechnol. 2008; 36(5):403-420; DOI: $10.1080 / 10731190802369755$.

19. EPA. Toxological review of barium and compounds. Cincinnati, OH: U.S Env Pro Agy. 2005; 1-57; CAS No. 7440-39-3.

20. Haug A. The affinity of some divalent metals to different types of alginates. Acta Chem Scand. 1961; 15:1794-1795; DOI: 10.3891/ACTA. CHEM.SCAND.15-1794. 